\title{
Semi-automated analysis of an optical ATP indicator in neural synapses
}

\author{
Taher Dehkharghanian $^{1 *}$, Arsalan Hashemiaghdam ${ }^{2 *}$, Ghazaleh Ashrafi ${ }^{2 \#}$ \\ ${ }^{1}$ Faculty of Health Science, McMaster University, Hamilton, On, Canada \\ ${ }^{2}$ Department of Cell Biology and Physiology, Department of Genetics, Needleman Center for \\ Neurometabolism and Axonal Therapeutics, Washington University School of Medicine in St. \\ Louis, St. Louis, MO, USA \\ * These authors contributed equally to this manuscript. \\ \# Corresponding author: E-mail: ghazaleh@wustl.edu
}

\begin{abstract}
Since its discovery in fireflies, bioluminescence has been used in a wide range of biological assays, from reporter gene assays to in vivo imaging. Bioluminescent light is produced from the enzymatic action of luciferase on its substrate luciferin using ATP as a cofactor. Recently, subcellular targeting of luciferase to neural synaptic vesicles has led to the development of the sensor Syn-ATP that allows for quantitative measurement of presynaptic ATP levels. Manual analysis of presynaptic bioluminescence signals from Syn-ATP is challenging due to signal heterogeneity and cellular motion in long imaging sessions. Here, we present a pipeline for semi-automated image analysis of Syn-ATP signals in the nerve terminals of hippocampal neurons. Our method streamlines data analysis and reduces user-introduced bias, thus enhancing the reproducibility and reliability of quantitative ATP imaging.
\end{abstract}

\section{Keywords}

Bioluminescence, synaptic vesicles, ATP, signal analysis

\section{Introduction}

In nature, living organisms as diverse as fireflies, copepods, and sea pansies produce natural light through bioluminescence. These organisms express the enzyme luciferase that emits visible light when it catalyzes the oxidation of its substrate luciferin powered by adenosine triphosphate (ATP). Bioluminescence imaging is a sensitive technique that relies on the detection of light emitted from the luciferase reaction. At saturating luciferin concentrations, luciferase light emission is proportional to ATP levels, thus luciferase can be used as a sensitive cellular ATP sensor. In contrast to fluorescence, where absorption of light of a certain wavelength results in emission at a longer wavelength, luminescence does not require excitation by light ${ }^{1}$. Therefore, bioluminescence imaging can be performed in the absence of any additional light sources and only requires a sensitive camera detector. Another advantage of bioluminescence as compared to fluorescence is its high sensitivity and low background signal. Fluorescence imaging of biological materials suffers from high level of autofluorescence, particularly in the green emission range, while this phenomenon is virtually absent in luminescence. In contrast, luminescence imaging of biological samples has low background noise which can be further minimized by proper light insulation of the experimental setup and sensitive detection systems. 
The first practical application of bioluminescence was the development of a reporter for gene expression using the North American firefly (Photinus pyralis) luciferase, which emits yellow-green light (emission peak at $557 \mathrm{~nm})^{2,3}$. Since then, novel mutant bioluminescent reporters emitting red light $(>600 \mathrm{~nm}$ ) have been engineered to improve tissue penetration for in vivo bioluminescence imaging (See Fig. 1a) ${ }^{4}$. Further modifications to luciferase thermostability and catalytic activity led to the development of ATP sensors for monitoring subcellular ATP levels ${ }^{5}$. For our experiments, we utilize a previously developed presynaptic ATP sensor, "Syn-ATP," to monitor ATP levels in the nerve terminals of cultured hippocampal neurons, especially during electrical activity. Syn-ATP is a genetically encoded optical reporter of ATP, available on Addgene (plasmid \# 51819; RRID: Addgene_51819) in which luciferase is targeted to synaptic vesicles through fusion with synaptophysin and additionally tagged with the fluorophore mCherry to normalize for reporter expression level (See Fig. 1b) ${ }^{6}$.

Since its development, Syn-ATP image analysis was performed manually with the software Image $\mathrm{J}$ and Microsoft Excel. In this method, the ImageJ Plugin Time Series Analyzer is used for selection of regions of interest (ROIs) corresponding to individual nerve terminals and measurement of fluorescence and luminescence signals over multiple time frames. This is followed by background subtraction and normalization of the luminescence signal of individual terminals to mCherry fluorescence to correct for variability in Syn-ATP expression and/or change in the focal plane. This method requires users to select the maximal number of mCherry-positive terminals in a recorded field while excluding cell bodies or large cellular clumps. Manual analysis is undesirable because it is time-consuming and subject to user bias. In addition, cellular motion and frame-to-frame movement of the selected data points in lengthy (several minutes) experiments complicates data analysis. To address the limitations of manual analysis, we have developed a semi-automated python-based analysis pipeline that measures Syn-ATP luminescence signals in individual neurons with appropriate background correction and normalization to fluorescence signal.

\section{Methods}

All animal experiments were performed with wild-type rats of the Sprague-Dawley strain in accordance with protocols approved by the IACUC at Washington University School of Medicine in St. Louis. Live imaging of rat hippocampal neurons was performed on a custom-built, inverted Olympus IX83 epifluorescence microscope equipped for luminescence and fluorescence imaging. Fluorescence excitation of mCherry was achieved with the TTL-controlled Cy3 channel of a Lumencor Aura III light engine. Both mCherry emission and Syn-ATP luminescence were directed through a Chroma 590nm long-pass filter and an Olympus UPlan Fluorite 40X 1.3 NA objective. Image acquisition was performed with an Andor iXon Ultra 897 camera, cooled to -95 ${ }^{\circ} \mathrm{C}$ to minimize the camera detection noise. Hippocampal neurons, plated on poly-ornithine coated coverslips, were mounted in a laminar flow perfusion chamber, maintained at $37^{\circ} \mathrm{C}$ with an OkoLab stage top incubator in Tyrode's buffer containing (in mM) $119 \mathrm{NaCl}, 2.5 \mathrm{KCl}, 2$ $\mathrm{CaCl}_{2}, 2 \mathrm{MgCl}_{2}, 50$ HEPES (pH 7.4), 2 D-Luciferin potassium salt (Gold Biotechnology), 1.25 lactate and 1.25 pyruvate, supplemented with $10 \mathrm{mM} \mathrm{6-cyano-7nitroquinoxalibe-2,} \mathrm{3-dione}$ (CNQX), and $50 \mathrm{mM}$ DL-2-amino-5phosphonovaleric acid (APV) to inhibit postsynaptic responses. Platinum-Iridium electrodes were used to evoke action potentials with $1 \mathrm{~ms}$ electrical pulses creating field potentials of $\sim 10 \mathrm{~V} / \mathrm{cm}$. For each data point, a 10-second fluorescence time 
lapse movie at $2 \mathrm{~Hz}$ imaging was collected, followed by a single luminescence image with 60 second exposure time. The alternating acquisition of fluorescence and luminescence images was repeated throughout the experiment. Unless otherwise indicated, all chemicals were obtained from Sigma-Aldrich. Image analysis was performed with Image J Time Series Analyzer (manual analysis) or the proposed semi-automated algorithm coded in python programming language, using Jupyter notebook, and scikit-learn machine learning library ${ }^{7,8}$. Data visualization and statistical analysis were performed in GraphPad Prism v9.0.

\section{Results}

We implemented unsupervised machine learning algorithms for our image analysis, particularly because a large and labeled dataset of hippocampal nerve terminals was not publicly available to implement supervised algorithms for ROI detection. Our analysis pipeline is composed of three main steps: background detection, cell body detection, and signal estimation. In order to improve consistency, the user is asked for verification and is allowed to modify the model's output for most steps of the process. Although our images have two channels (mCherry fluorescence and luminescence), we used the luminescence channel for background and cell body detection due to its low background signal. We then applied onto fluorescence images the background and cell body masks obtained from the luminescence channel.

\section{Development of analysis pipeline}

The first step of the proposed pipeline was to distinguish neurons from the background. The input image of size $512 \times 512$ pixels was down-sampled to $128 \times 128$ pixels, divided into regions of $4 \times 4$ pixels, and each region was represented by the median of its pixel values. This step was taken to limit outlier effects and to reduce the dimensions of the clustering problem. Next, kmeans clustering from scikit-learn python library were used to cluster regions into two groups ${ }^{8,9}$. Pixel intensities were transformed into a one-dimensional vector of size $16,384(128 \times 128)$. This vector was given to the algorithm to produce two clusters, one corresponding to the image background, and the other to the regions of interest. Since K-means is an unsupervised machine learning algorithm, it does not require any training before being applied on the data. It was observed that this clustering scheme accurately distinguished background from the regions of interest (see Fig. 2a). We used luminescence images to create background masks and applied the same masks on fluorescence images (See Fig. 2b). Background signal in each channel was then calculated as the average intensity of the background pixels.

The second step was to detect the neural cell body expressing the Syn-ATP reporter and to exclude it from further analysis as energy metabolism in this compartment may be different from nerve terminals where our studies are focused on. To this end, the user is first asked to set the custom width (d) of the cell body or use the default preset value of 32 pixels. Then, a square of size $d x d$ pixels is moved one pixel at a time, and the mean pixel intensity was calculated for each region. The region with the highest mean intensity was deemed to be the cell body (see Fig. 2a). The shape and size of the cell body were not consistent across our neuronal samples, and in some cases, a cell body was not captured in our images. To address this issue, the user is asked to provide their input determining whether the cell body was accurately detected. If not, 
the user has the option to manually set a custom-sized bounding box to cover the cell body. Once detected, the cell body is then omitted from further analysis.

Once masking was performed, total signal intensity in each channel was calculated from which background intensity was subtracted to yield net luminescence and fluorescence intensities. This process was iterated 8 times in accordance with the number of imaging time points. Then, average presynaptic ATP level in each neuron (in arbitrary units) was calculated as such:

$\mathrm{L} / \mathrm{F}=$ (Luminescence- Background)/ (Fluorescence - Background)]

The absolute $L / F$ value is dependent on image acquisition parameters that may need to be modified during a project. For modifications that produce a linear change in signal intensity, such as adjustments to camera EM gain settings, we have introduced a simple true/false argument to adjust calculations of ATP levels by a coefficient factor.

\section{Performance Evaluation}

To evaluate the performance of our analysis pipeline, raw fluorescence and luminescence images from neuronal samples $(n=26)$ were analyzed both manually and with our semiautomated tool. The experiment consisted of four baseline 1-minute timepoints, followed by 1 minute of electrical stimulation at $10 \mathrm{~Hz}$ applied between timepoints 4 and 5 . The neurons were imaged for three additional timepoints after stimulation. Compared to manual analysis, our semi-automatic analysis method generated higher L/F values. We speculate that this is due to its more precise determination of background signal values which were lower than when manually selected (Fig. 3a).

We then assessed consistency of the measurements obtained from semi-automated pipeline compared to manual analysis. In our experiments, the timepoints prior to stimulation represent baseline ATP levels and are expected to have minimal variation. We calculated the L/F values of the three initial timepoints using both methods and determined the measurement variability $(\Delta \mathrm{L} / \mathrm{F})$ among them. The semi-automated approach significantly decreased measurement variabilities thus increasing the reliability of Syn-ATP analysis ( $p$-value $=0.0014$ ). The variation among the three baseline data points across cells $(n=26)$ was $3.4+/-0.9 \%$ versus $6.8+/-0.6 \%$ in semi-automated and manual analysis, respectively (see Fig. 3c). These findings confirmed that our code increases consistency and minimizes sampling biases in ROI and background detection. It has been well documented that acidification of the cytosol during electrical stimulation causes a reduction in $L / F$ value that is due to a decrease in luciferase enzymatic activity as its pKa of 7.03 is close to the cytosolic pKa of $6.8^{6}$. Indeed, both manual and semiautomated analysis methods detected similar acidification effects on L/F values that were normalized to the baseline (Fig. 3c).

To assess the validity of our method, we compared the variation of measured intensity between two neural populations ( $n_{1}=26, n_{2}=10$ cells) with different baseline ATP levels and Syn-ATP L/F values. We expected to observe a similar level of change in L/F between the two groups using either method. We calculated $\Delta(\Delta \mathrm{L} / \mathrm{F})$ for each cell population and compared both datasets. Unpaired t-test with Welch's correction reported no significant change in inter-population intensity differences by either analysis methods ( $p$-value $=0.1418)$. Therefore, we conclude that 
our semi-automated approach has the same validity as manual analysis, and it does not alter observed differences in Syn-ATP L/F values (See Fig. 3d).

\section{Conclusion}

Syn-ATP represents a powerful and robust application of the luciferase reaction for measurement of presynaptic ATP levels. However, analysis of Syn-ATP data is challenging and signal variability in the raw images, and the potential for user selection bias. Standardizing this process with a unified approach would minimize inconsistencies in data analysis. Here, we propose a semi-automated pipeline that facilitates signal analysis for two-channel images of fluorescence and luminescence. First, the tool helps the user to determine background signals in both channels in an unbiased manner. Second, in the case of neurons, it enables the user to mask specified regions such as the cell body. In imaging sessions that last for several minutes, axonal movement shifts the position of nerve terminals in the field of view and poses challenges for manual tracking of several data points in an image stack. Our approach would circumvent this problem by analyzing the entire image rather than individual user-selected ROls that correspond to individual nerve terminals. We acknowledge that our semi-automated approach also has limitations. Specifically, our pipeline does not distinguish between ATP signals originating from nerve terminals versus signals from axonal regions spanning terminals or other neuronal processes where Syn-ATP may be mistargeted. We have also observed that sudden changes in the background signal interfere with the code and produce unreliable results. Our future endeavors with expanded datasets would be directed towards resolving these hurdles.

In summary, we have developed a semi-automated pipeline for analysis of dual fluorescence/bioluminescence imaging of ATP in neural synapses. Our approach utilizes the entire field of view for data analysis thereby removing user sampling bias. It also standardizes background signal measurement and reduces and variability. The code is publicly available on the GitHub library (https://github.com/ashrafilab/SynATP-Analysis) and can be modified for customized bioluminescence imaging experiments.

\section{Contributions}

T.D. and A.H. share co-first authorship. G.A., A.H., and T.D. conceptualized, designed the method, and wrote the manuscript. T.D. wrote the code in Python. G.A. and A.H. performed experiments and collected the data. G.A. is the corresponding author and supervised the correspondence.

\section{Ethics declarations}

\section{Competing interests}

The authors declare no competing interests.

\section{Acknowledgments:}

The authors would like to thank Javid Dadashkarimi for proofreading the manuscript. Figure 1 was prepared using the BioRender software (Biorender.com).

\section{Biographies:}


- Ghazaleh Ashrafi studied cell biology at the University of Alberta in Canada and obtained her Ph.D. from Harvard University and completed her postdoctoral fellowship at Weill Cornell Medical College studying the regulation of energy metabolism in active synapses. Since January 2020, she is an Assistant Professor in the Department of Cell Biology and Physiology, and Genetics at Washington University School of Medicine in St. Louis and her laboratory studies neuronal metabolic adaptations to energetic stress.

- Arsalan Hashemiaghdam completed his degree in Medicine from Tehran University of Medical Sciences in Iran and continued his research endeavors at Massachusetts General Hospital where he studied the role of fatty acids and ER stress in glioblastoma. Afterward, he moved to Yale University to investigate the role of microglia in the early stages of neurodegeneration. He joined the Ashrafi lab in 2020 to study regulatory mechanisms of mitochondrial ATP production in nerve terminals.

- Taher Dehkharghanian received his medical degree from Tehran University of Medical Sciences in Iran and pursued a master's degree in computer science from Ontario Tech, Canada. His area of research is in the intersection of artificial intelligence and medical imaging. He joined McMaster University as a postdoctoral research fellow in 2021.

\section{Code, Data, and Materials Availability}

The image analysis code is available at Ashrafi Lab's GitHub repository.

(https://github.com/ashrafilab/SynATP-Analysis)

\section{References}

1 Badr CE, Tannous BA. Bioluminescence imaging: Progress and applications. Trends Biotechnol 2011:624-33.

2 JR de W, KV W, M D, DR H, S S. Firefly luciferase gene: structure and expression in mammalian cells. Mol Cell Biol 1987;7:725-37.

3 Branchini BR, Ablamsky DM, Murtiashaw MH, Uzasci L, Fraga H, Southworth TL. Thermostable red and green light-producing firefly luciferase mutants for bioluminescent reporter applications. Anal Biochem 2007;361:253-62.

4 Branchini BR, Ablamsky DM, Davis AL, Southworth TL, Butler B, Fan F, et al. Redemitting luciferases for bioluminescence reporter and imaging applications. Anal Biochem 2010;396:290-7.

5 Jain P, Neveu B, Velot L, Wu L, Fradet Y, Pouliot F. Bioluminescence microscopy as a method to measure single cell androgen receptor activity heterogeneous responses to antiandrogens. Sci Rep 2016;6:.

6 Rangaraju V, Calloway N, Ryan TA. Activity-driven local ATP synthesis is required for synaptic function. Cell 2014;156:825-35.

7 Kluyver T, Ragan-Kelley B, Pérez F, Granger BE, Bussonnier M, Frederic J, et al. Jupyter Notebooks-a publishing format for reproducible computational workflows. vol. 2016. 2016.

8 Pedregosa F, Varoquaux G, Gramfort A, Michel V, Thirion B, Grisel O, et al. Scikit-learn: Machine learning in Python. J Mach Learn Res 2011;12:2825-30.

$9 \quad$ Lloyd S. Least squares quantization in PCM. IEEE Trans Inf Theory 1982;28:129-37.

Figures: 


\section{Figure 1}

a Luciferase + Luciferin $+\mathrm{ATP} \stackrel{\mathrm{Mg}^{2+}}{\longrightarrow}$ Luciferase-Luciferyl-AMP $+\mathrm{PP}_{\mathrm{i}}$
Luciferase-Luciferyl-AMP $+\mathrm{O} 2 \longrightarrow$ Luciferase + Oxyluciferin $+\mathrm{AMP}+\mathrm{CO} 2+\mathrm{hv}$

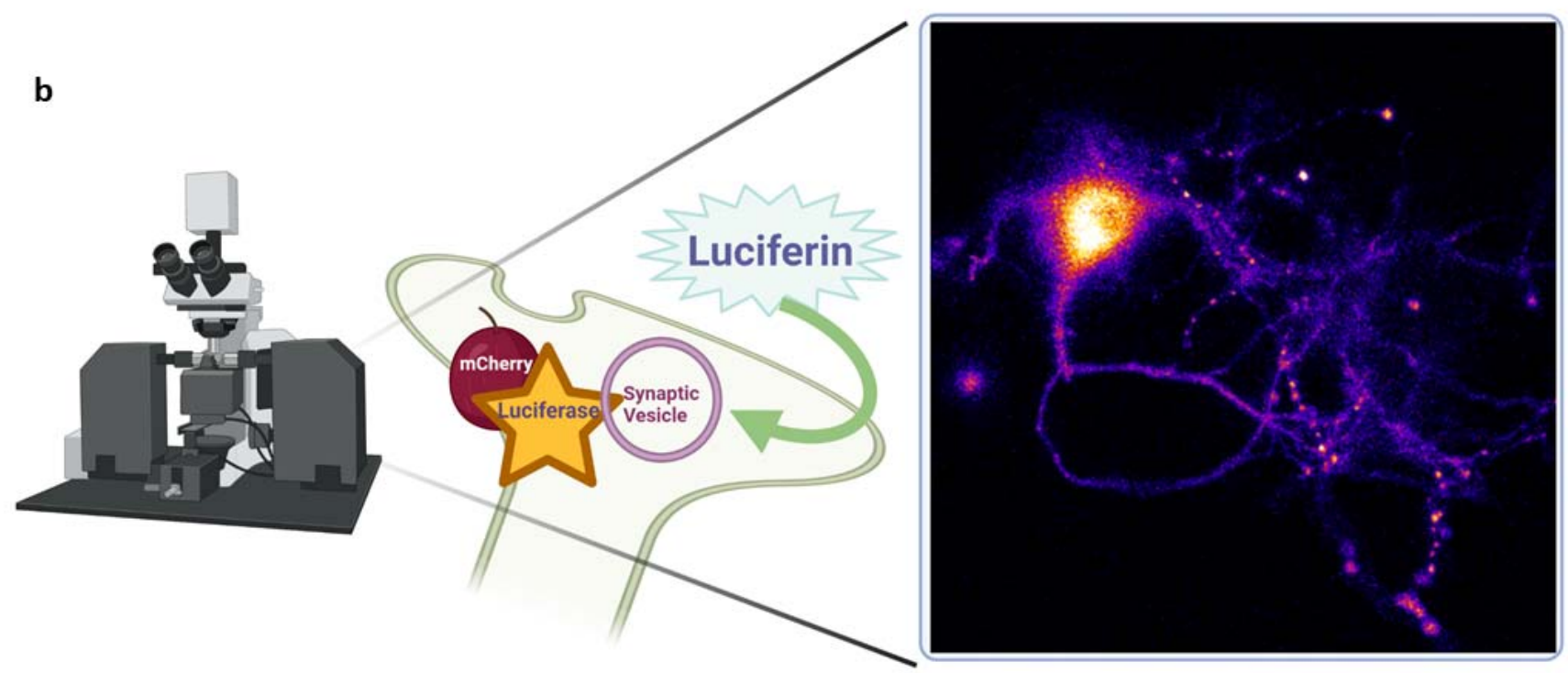

Schematic of ATP bioluminescence imaging in nerve terminals. a) The bioluminescence chemical reaction. b) Dual fluorescence and luminescence imaging of hippocampal nerve terminals expressing Syn-ATP in dissociated cultures is used for quantitative assessment of presynaptic ATP level. 


\section{Figure 2}

a

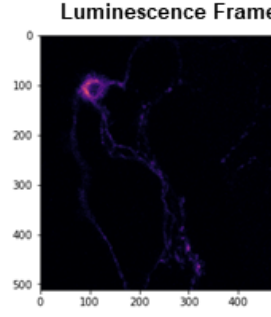

b

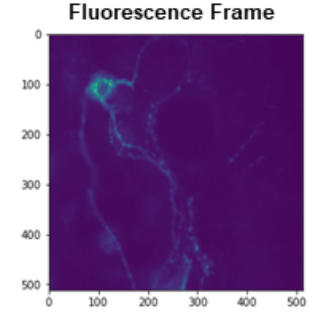

Downsampled Image

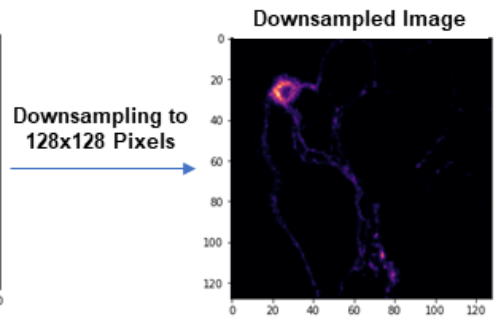

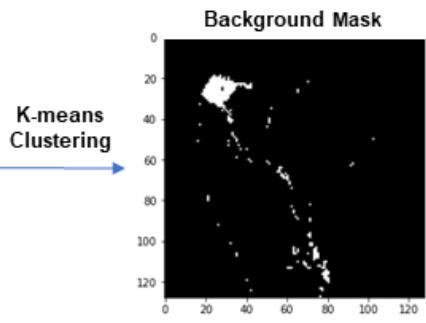
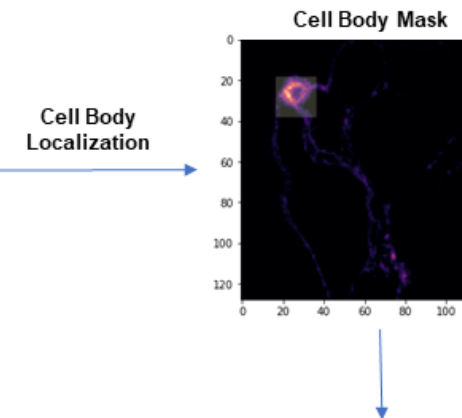

Downsample and Apply Luminescence Masks on the Fluorescence Frame
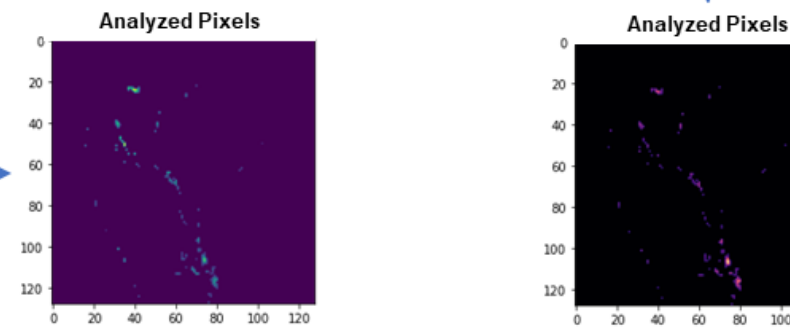

An Image analysis pipeline for background signal determination and cell body removal. a) Luminescence image was downsampled from $512 \times 512$ pixels to $128 \times 128$ pixels. K-means clustering algorithm was implemented on pixel values to produce two clusters in order to create the background mask. Next, the region with the highest total signal intensity was detected and deemed as the cell body. Both background and cell body were removed from further analysis. b) Background and cell body masks generated from the luminescence image were applied on the fluorescence channel. 


\section{Figure 3}

a

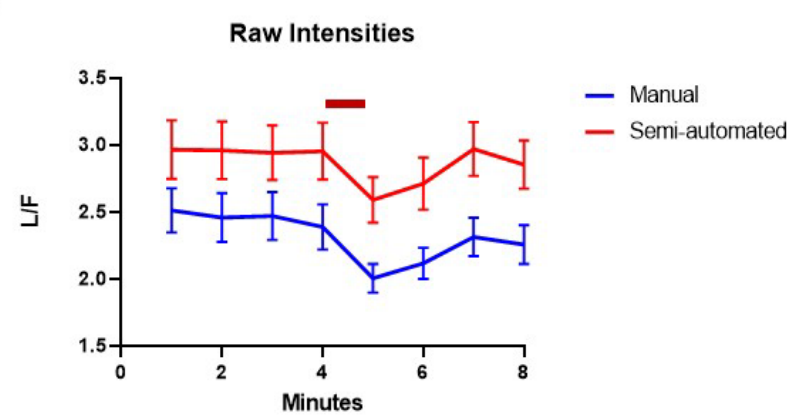

b

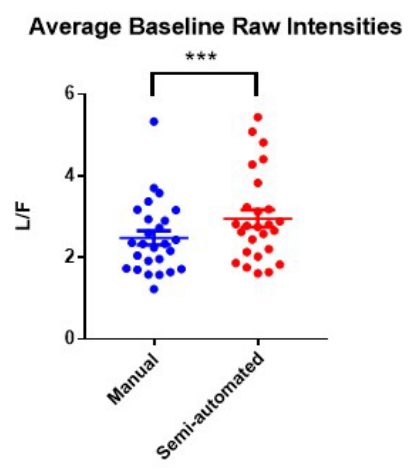

d

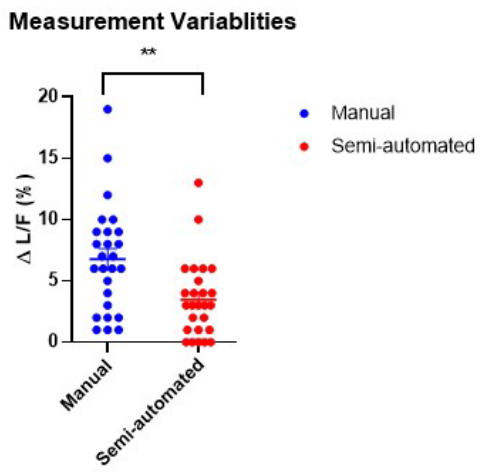

Measurement Validity Between Methods

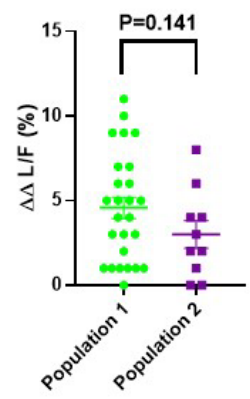

- Population

- Population

Quantitative comparison of Syn-ATP image analysis by manual and semi-automated methods. Hippocampal neurons expressing Syn-ATP $(n=26)$ were imaged for 8 minutes, electrically stimulated for $1 \mathrm{~min}$ at $10 \mathrm{~Hz}$ frequency (crimson bar) and analyzed by either method. a) Average traces of presynaptic luminescence values normalized by fluorescence intensity values ( $L / F)$. ( $n=52$ neurons) b) Baseline pre-stimulation $L / F$ values were significantly different between manual and semi-automated methods (paired T-test: $t=3.948, d f=25, p=0.0006$ ). $c$ ) $L / F$ traces normalized to pre-stimulation baseline, analyzed by either method, show similar patterns. d) Measurement variability of pre-stimulus L/F values was determined as \% deviation from the mean $(\Delta \mathrm{L} / \mathrm{F})$. Semi-automated analysis significantly reduces variability (paired $t$-test: $t=3.584$, $\mathrm{df}=25, \mathrm{p}=0.014)$. e) Measurement validity of the analysis methods was assessed by comparing two distinct neural populations with different baseline ATP levels ( $P 1, n_{1}=26, P 2, n_{2}=10$ ). First, $\Delta \mathrm{L} / \mathrm{F}$ in each population was calculated. Measurement variability between two methods for each cell population was defined as the \% difference of $\Delta \mathrm{L} / \mathrm{F}(\Delta \Delta \mathrm{L} / \mathrm{F}) . \Delta \Delta \mathrm{L} / \mathrm{F}$ was not significantly different between the two cell populations, indicating that the semi-automated approach is as valid as conventional manual analysis (unpaired $t$-test: $t=1.529, \mathrm{df}=20.33, \mathrm{p}=0.141$ ). 